\title{
Regional Evaluation of GOCE-Based GGMs with Ground-Based Gravity and GPS/Levelling Data over Egypt
}

\author{
Ahmed Saadon1, Basem Elsaka ${ }^{2,3}$, Mohamed El-Ashquer1, Gamal El-Fiky ${ }^{1,4}$ \\ ${ }^{1}$ Construction Engineering and Utilities Department, Faculty of Engineering, Zagazig University, Zagazig, Egypt \\ ${ }^{2}$ National Research Institute of Astronomy and Geophysics (NRIAG), Cairo, Egypt \\ ${ }^{3}$ Institute of Geodesy and Geoinformation, University of Bonn, Bonn, Germany \\ ${ }^{4}$ The Bilbies Higher Institute of Engineering, Bilbies, Egypt \\ Email: eng.ahmedsaadon@gmail.com
}

How to cite this paper: Saadon, A., Elsaka, B., El-Ashquer, M. and El-Fiky, G. (2019) Regional Evaluation of GOCE-Based GGMs with Ground-Based Gravity and GPS/Levelling Data over Egypt. International Journal of Geosciences, 10, 652-668.

https://doi.org/10.4236/ijg.2019.106037

Received: May 10, 2019

Accepted: June 25, 2019

Published: June 28, 2019

Copyright (c) 2019 by author(s) and Scientific Research Publishing Inc. This work is licensed under the Creative Commons Attribution-NonCommercial International License (CC BY-NC 4.0). http://creativecommons.org/licenses/by-nc/4.0/

\section{Open Access}

\begin{abstract}
The gravity field and steady-state ocean circulation explorer (GOCE) satellite mission has provided numerous Global Geopotential Models (GGMs) with different processing methodologies and model accuracies. In the current contribution, the latest releases of GOCE-based GGMs are evaluated on the regional scale using the available terrestrial GPS/Levelling and gravity data collected over Egypt. To overcome the spectral inconsistency between the GOCE-based GGMs and the ground-based data, the spectral enhancement method (SEM) is applied. Five of GOCE-based GGMs have been used, namely GOSG01S, IGGT_R1, IfE_GOCE05s_GO_CONS_GCF_2_SPW_R5 (SPW_R5 in the following) and NULP-02. The evaluation process of GOCE-based GGMs with the available ground data over Egypt considering the SEM method shows remarkable improvements obtained from the SPW_R5 model. The model provides lower differences of the standard deviations with respect to the EGM2008 and the other applied geopotential gravity models as well as the applied ground-based gravity and GPS/Levelling data. The findings regarding the ground-based data show obvious reductions of about $15.16 \%$ and $32.22 \%$ achieved by the GOCE-based model in term of standard deviations differences of gravity anomalies and geoid heights, respectively. Therefore, the SPW_R5 model is recommended to be applied as a reference model for compensating the long-to-short wavelength (up to spherical harmonics degree/order 280) components when modelling the gravimetric geoid over Egypt.
\end{abstract}

\section{Keywords}

GOCE-Based GGMs, Gravity Anomalies, GNSS/Levelling, Spectral 
Enhancement Method (SEM)

\section{Introduction}

The Global Geopotential Models (GGMs) derived from the GOCE Gradiometry measurements led to improvement of the knowledge of the gravity field of the Earth, especially for the medium-to-short wavelength spectrum which plays a main component in geoid determination. One has to mention here that the GOCE mission was launched on March 2009 until October 2013 by the European space Agency (ESA) from the Plesetsk Cosmodrome in Russia into space. One of its main goals was to deliver the accuracy of the geoid heights and gravity anomalies to about $1-2 \mathrm{~cm}$ and $1 \mathrm{mGal}\left(1 \mathrm{mGal}=1.00 \times 10-5 \mathrm{~m} \cdot \mathrm{s}^{-2}\right)$, respectively. These accuracies have been successfully achieved at spatial resolution of about $100-\mathrm{km}$ which coincides approximately to spherical harmonics degree and order ( $\mathrm{SH} \mathrm{d} / \mathrm{o}) 200$. In addition, different research teams worked on processing the data collected from the GOCE mission to provide different gravity field solutions. Among these solutions, three types of GOCE-based GGMs have been established by three processing techniques: time wise (TIM) [1], direct (DIR) [2] and space wise (SPW) [3] which are developed by the European space Agency (ESA) project called GOCE High-Level Processing facility (HPF) [4]. Moreover, GOCE Gradiometry data have been combined with other satellite-based data to develop the GGMs such as GOCO02s till GOCO05c, EIGEN-6C \& S, GGM05C \& $\mathrm{G}$ and GOGRA02s \& 04s.

For examining the performance of numerous GOCE-based geopotential models, gravity field functional (here gravity anomalies and geoid heights) should be evaluated using ground-based datasets. This helps obviously to assess the accuracy of GOCE-based GGMs.

The main aim of this study is evaluate the fifth generation of GOCE-based satellite over Egypt using the available ground-based gravity field anomalies and GPS/Levelling geoid heights. Five of these models are evaluated (GOSG01S, IGGT_R1, IfE_GOCE05s_GO_CONS_GCF_2_SPW_R5 and NULP-02). In addition, the gravity data used in the current study are investigated for first time to approximate the gravity field over Egypt. This allows us accordingly to select the most suitable global model which best approximates the gravity field over the Egyptian region.

We should note here that among various evaluation procedures of satellite-based models using ground-based data, we apply the spectral enhancement method [5]. Thus the geopotential model that will provide best statistical fits in terms of least standard deviations (Std) differences with respect to the observed ground data would be assumed to be the most suitable model adopted for the determination of gravimetric geoid in Egypt, which is the scope of our further investigations. 
It should be noted here that GOCE-based GGMs have been validated over different regions of the world by different research studies, e.g. ([5]-[15]). [15] Investigated the contribution of the fourth and fifth released GOCE-based GGMs (DIR 4, 5; TIM 4, 5, SPW 4) in improvements of geoid model in the long-wavelength components over Egypt. They found that the GOCE-based SPW_R4 (up to SH d/o 200) is the most suitable global model that could be used in modelling the local geoid over Egypt. However, in the current study, we would examine the latest releases of GOCE-based GGMs beyond SPW_R4 with new gravity data used for the first time that would provide further improvements of the gravity and geoid approximations over Egypt.

This paper is organized as follows: Section 2 reviews briefly the datasets used in this evaluation study. Section 3 introduces the validation strategy using GOCE-based GGMs to improve the geoid height determination on a regional scales (over Egypt). In the first step, GOCE-based GGMs are compared with the Earth Global Model 2008 (EGM2008) [16]. In the second step, a comparison between ground-based geoid heights is carried out. In the later comparison, the remaining short wavelength, e.g., beyond maximum d/o of GOCE-based GGMs components of geoid heights will be compensated using the EGM2008 at medium and high frequency (i.e., up to $\mathrm{d} / \mathrm{o} 2190$ ) in addition to the high and very high frequency (i.e., beyond d/o 2190), induced from local topography information which is considered as a remaining omission error. The results are discussed in Section 4. Finally, conclusions are provided in Section 5.

\section{Datasets}

The datasets used in this study are divided into three main categories; 1) GOCE-based satellite only models and the EGM2008 which is used in the current study as a reference model, 2) ground-based data in the sense of terrestrial free-air gravity anomalies and GPS/Levelling data collected over the Egyptian region; and 3) High resolution topographic data from the SRTM30_PLUS (Shuttle Radar Topography Mission) digital terrain model. In the following sections, a brief description of each dataset will be given.

\subsection{Satellite-Based Geopotential Models (GOCE and the EGM2008)}

In the period between 2010 and 2014 several GOCE-based geopotential models differing in their processing strategies and time span of observations have been released. Among of them, we use five of the latest releases of GOCE-based GGMs. Based on the fifth generation of the different processing techniques. These models are provided publically by the International Centre for Global Earth Models (ICGEMs) in terms of geopotential spherical harmonic coefficients (icgem.gfz-potsdam.de/ICGEM/). Table 1 shows a summary of each model characteristics used in this study. Since the GOCE-based GGMs are limited by a finite spatial resolution, the EGM2008 has been used as a reference gravitational model in order to complete the short wavelength beyond the maximum spherical 
Table 1. A summary of GOCE-based GGMs applied within this investigation.

\begin{tabular}{ccccc}
\hline Model name & Year & Maximum degree & Data & References \\
\hline NULP-02s & 2017 & 250 & GOCE (42 months $)$ & {$[18]$} \\
SPW_R5 & 2017 & 330 & GOCE (42 months $)$ & {$[19]$} \\
IfE_GOCE05s & 2017 & 250 & GOCE (42 months $)$ & {$[20]$} \\
IGGT_R1 & 2017 & 240 & GOCE (2 months $)$ & {$[21]$} \\
GOSG01S & 2018 & 220 & GOCE (20 months $)$ & {$[22]$} \\
\hline
\end{tabular}

harmonics degree of the GOCE-based models. The EGM2008 has been developed by the USA National Geospatial-Intelligence Agency (NGA) completed to d/o 2160 with some additional terms up to d/o 2190 [16] with a spatial resolution of about $9 \mathrm{~km}$. One should note here that the difference between the ground-based geoid heights and the corresponding ones of the EGM2008 is about $54 \mathrm{~cm}$ over the Egyptian region (see [15]). Therefore, we believe that in such regions of poor or heterogeneously data distribution, GOCE-based GGMs may improve the determination/modeling of the regional geoid heights.

\subsection{Ground-Based Datasets}

\subsubsection{Free-Air Gravity Field Anomalies}

In this study, 85 points in terms of free-air gravity field anomalies have been used. Figure 1 shows the distribution of the terrestrial gravity dataset over a topographic map of Egypt. The first-order Egyptian national gravity networks (ENGSN97) have been collected by the Survey Research Institute (SRI) in different projects over the last 5 years. The accuracy of ENGSN97 gravity values is about $0.022 \mathrm{mGal}$. Despite the sparse distribution of the gravity data, one may use them in the evaluation study since the data almost cover the whole territory.

\subsubsection{The Available GPS/Levelling Dataset}

The GPS/Levelling dataset was provided by the Egyptian Survey Authority from the Egyptian National High Accuracy Reference Network (HARN) consists of 30 stations. However, 13 of them (located in remote areas) have no observed orthometric heights and consequently, no undulations could be obtained for these stations. So only 17 GPS stations (see Figure 1) observed by the Egyptian Survey Authority to form the New Egyptian Datum 1995 (NED-95) are used in our study. In this network, the GPS observations were tied to some of the International Geodetic Stations (IGS) reference system [17]. It is believed that the HARN GPS network is the most fitted GPS framework in Egypt because of the precise instruments used, the connection to the IGS Stations, the use of precise satellite ephemerides and the utilization of accurate processing and adjustment software. The accuracy of the HARN network is 0.1 parts per million (ppm). This GPS/Levelling data are the only available dataset in the region in our investigation and, thus, have been used as it is. 


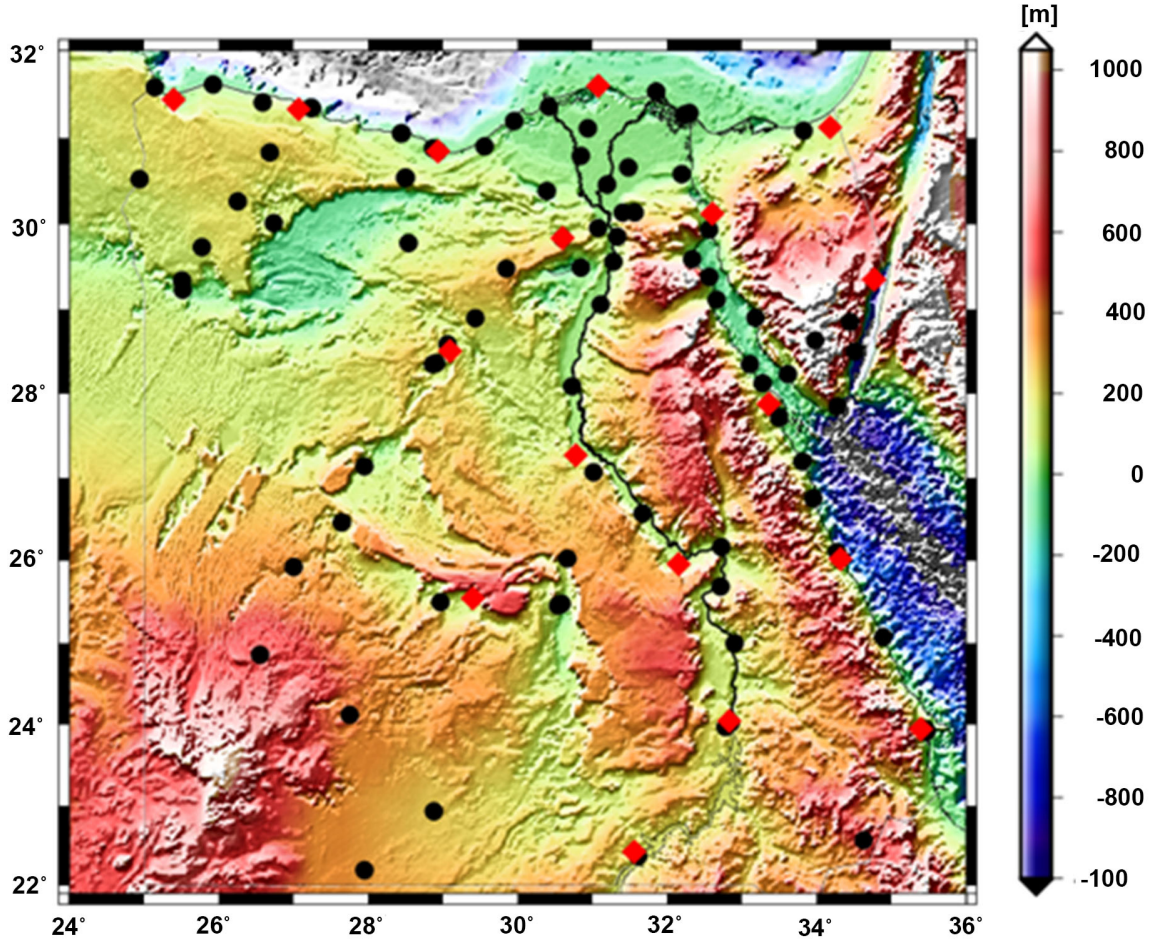

Figure 1. Distribution of the 85 gravity stations from SRI network (black) and 17 GPS/ Levelling benchmarks (red) in Egypt.

\subsection{Topography Data}

The SRTM30_PLUS (Shuttle Radar Topography Mission of spatial resolution about $\sim 900 \mathrm{~m}, 30$ arc-sec) data are used in this study to compute the topographic potential effect over Egypt region. The SRTM30_PLUS has been provided by the institution of Oceanography, University of California. The detailed information concerning the development of the SRTM30_PLUS are given in the website http://topex.ucsd.edu/WWW_html/srtm30_plus.html. The topographic heights as provided from the SRTM " $30 \times 30$ " for the Egyptian region between latitude 22 - 32 and longitude $24-35$ are ranging between $-3195 \mathrm{~m}$ and $2453 \mathrm{~m}$. Since the majority of the data (away from the Mediterranean Sea and the Red Sea) are between $-160-1390 \mathrm{~m}$, we have limited the map scale of Figure 1 up to \pm 1000 $\mathrm{m}$.

\section{Evaluation Strategy}

In the following, the evaluation of five of the latest GOCE-based GGM releases is investigated by two main approaches. At first, validation of the five investigated GOCE-based GGMs with the EGM2008 as a reference model in our study at a regional scale over Egypt is considered. We should note here that our selection of the EGM2008 is based on the fact that it doesn't include satellite data into its solution, and hence, a fair judgment can be concluded to find out which of the five GOCE-based models approximate the gravity field well over Egypt. In order to perform such validation on a regional scale, the spectral inconsistency be- 
tween GOCE-based models and the EGM2008 must be taken into consideration since they differ in resolution. Therefore, the solution in terms of spherical harmonics coefficients $(\mathrm{SH})$ of each model has been restricted from $\mathrm{SH}$ d/o 2 up to $\mathrm{Nmax}=100,110, \cdots, 320,330(10 \mathrm{~d} / \mathrm{o}$ step $)$ on grids from $1.8^{\circ} \times 1.8^{\circ}, 1.64^{\circ} \times$ $1.64^{\circ}, \cdots, 0.56^{\circ} \times 0.56^{\circ}, 0.55^{\circ} \times 0.55^{\circ}$, respectively. Second type of validation is performed using the available terrestrial gravity data and GPS/Levelling height over Egypt, where the free air gravity anomalies and geoid heights obtained from GOCE-based models are compared with the corresponding ones obtain from terrestrial data. For this type, the spectral enhancement method (SEM) has been applied to overcome the inconsistency of spectral contents in such a way that the maximum $\mathrm{SH} d$ /o of GOCE-based model will be completed with the reference model (EGM2008) up to degree 2190 and the gravity signal beyond 2190 will be added from the residual terrain modelling which are extracted from the topographic data (see Section 2.3). To estimate the residual terrain, the Terrain Correction (TC) program developed by [23] has been used (see Section 3.2).

\subsection{Validation with the EGM2008}

\subsubsection{Spectral Validation on the Global Scale}

First, we are interested here to compare the medium-to-short spectrum that GOCE-based GGMs provides with the corresponding range given by the EGM2008. The comparison is given in terms of degree variances which represent the power of signal at various spectral wavelengths [24]. They can be calculated by the following formula:

$$
\sigma_{n}^{2}=\sum_{m=0}^{n}\left(c_{n m}^{2}+s_{n m}^{2}\right)
$$

Second, the error degree variances express the total error power at various spectral wavelengths. They can be calculated by the following formula:

$$
\sigma_{n}^{\prime 2}=\sum_{m=0}^{n}\left(\sigma_{c_{n m}}^{2}+\sigma_{s_{n m}}^{2}\right)
$$

Using the reference model (EGM2008), the different degree variance and difference error degree variance of the GOCE-based GGMs can be calculated for any potential functional, as geoid height and gravity anomaly, by multiplying Equation (1) and/or Equation (2) by the proper eigenvalue (see Table 2). This provides the spectral linkage between the different functional of the disturbing potential at different altitudes on the Earth's surface [25].

Table 2. The eigenvalue for some potential functional [25].

\begin{tabular}{ccc}
\hline Potential function & Eigenvalue & Unit \\
\hline Signal & 1 & Dimensionless \\
Geoid height $(\mathrm{N})$ & $\mathrm{R}$ & $\mathrm{M}$ \\
Gravity anomaly $(\Delta \mathrm{g})$ & $\frac{G M}{R^{2}}(n-1) 10^{5}$ & $\mathrm{mGal}$ \\
\hline
\end{tabular}


The difference degree variances, which are defined for each degree (i.e. they are dependent on the degree not the order) between the GOCE-based GGMs and the EGM2008 in terms of geoid heights, read

$$
\Delta \sigma_{n}=R_{E} \sqrt{\sum_{m=0}^{n}\left(\Delta C_{n m}^{2}+\Delta S_{n m}^{2}\right)}
$$

With

$$
\Delta C_{n m}=C_{n m}^{G O C E \text { model }}-C_{n m}^{E G M 08} \text { and } \Delta S_{n m}=S_{n m}^{G O C E \text { model }}-S_{n m}^{E G M 08} \text {. }
$$

However, for reliable comparison and interpretation as well, it is recommended to use a scalar quantity which characterizes the commissioning error of a gravity coefficients signal, therefore the cumulative geoid error is used:

$$
\Delta \sigma_{\text {cum }}=R_{E} \sqrt{\sum_{n=2}^{n_{\max }} \sum_{m=0}^{n}\left(\Delta C_{n m}^{2}+\Delta S_{n m}^{2}\right)}
$$

Regarding gravity anomalies (in mgal), the difference degree variances read:

$$
\sigma_{n}=\frac{G M}{R_{E}^{2}}(n-1) 10^{5} \sqrt{\sum_{m=0}^{n}\left(\Delta C_{n m}^{2}+\Delta S_{n m}^{2}\right)}
$$

And their corresponding cumulative errors read

$$
\Delta \sigma_{\text {cum }}=\frac{G M}{R_{E}^{2}}(n-1) 10^{5} \sqrt{\sum_{n=2}^{n_{\max }} \sum_{m=0}^{n}\left(\Delta C_{n m}^{2}+\Delta S_{n m}^{2}\right)}
$$

Where $R_{E}$ is the mean radius of the Earth at the reference ellipsoid ( $\approx 6378.137$ $\mathrm{km}), G$ is the geocentric gravitational constant, $M$ is the mass of the Earth ( $G M \approx 0.3986004415 \times 10^{15}$ ), $n$ and $m$ are the degree and order (d/o) of model, respectively.

\subsubsection{Validation with the EGM2008 on the Regional Scale}

On the regional scale, the GOCE-based GGMs have been evaluated with the EGM2008 over the study area of Egypt. The GOCE-based GGMs used in this investigation differ in their spectral resolution and the methodology of processing data. As a result, the spectral consistency of each compared model has to be taken into consideration toward an effective comparison. Therefore, each model has been bounded from SH d/o 2 up to $\mathrm{Nmax}=100,110, \cdots, 320,330$ $\left(10 \mathrm{~d} / \mathrm{o}\right.$ step) on grids from $1.8^{\circ} \times 1.8^{\circ}, 1.64^{\circ} \times 1.64^{\circ}, \cdots, 0.56^{\circ} \times 0.56^{\circ}, 0.55^{\circ} \times$ $0.55^{\circ}$, respectively. The evaluation has been performed in terms of difference of gravity anomalies $(\delta \Delta g)$ Equation (7) and geoid heights $(\delta \Delta N)$ Equation (8) obtained from GOCE-based models and the corresponding ones from the EGM2008 as:

$$
\begin{aligned}
& \delta \Delta g=\left.\Delta g_{G O C E(\text { grid })}\right|_{2} ^{N_{\max }}-\left.\Delta g_{E G M 2008(\text { grid })}\right|_{2} ^{N_{\max }} \\
& \delta \Delta N=\left.\Delta N_{G O C E(\text { grid) }}\right|_{2} ^{N_{\max }}-\left.\Delta N_{E G M 2008 \text { (grid) }}\right|_{2} ^{N_{\max }}
\end{aligned}
$$

\subsection{Validation with Ground Data over Egypt}

The use of ground-based datasets such as terrestrial gravity and GPS/Levelling is quite important to obtain a reliable external validation and accuracy assessment of the investigated GOCE-based GGMs, However, the difference in spectral do- 
mains between the GOCE-based GGMs and the ground data is a big issue that must be taken into account. This is back to the fact that the GOCE-based GGMs are limited to a finite maximum $\mathrm{SH} \mathrm{d} / \mathrm{o}$, whereas the terrestrial data have theoretically the full spectra, particularly the short and very short spectra which are not included in the GGMs as shown in Figure 2. Numerous approaches have been developed in order to overcome this issue, e.g. the spectral enhancement method (SEM) ([5] [26]) and the use of Gaussian "Low-Pass" filtering [27]. The latter approach has many techniques such as Gaussian filters, spectral filters, least-squares collocation (LSC) or spherical-harmonic synthesis (SHS). They may be a beneficial approach when the terrestrial ground data are available in some suitable arrangements, for instance, profiles, grids or at densely scattered locations [28]. On the other hand, the SEM approach can be used for GGMs validation independent on the spatial distribution of the terrestrial data. This is a significant advantage when using irregularly distributed ground observations such as our study case here (Egypt) which suffer from poor terrestrial gravity and GPS/Levelling data. Consequently, the SEM will be used in this investigation.

The basic idea of the SEM to validate the GOCE-based GGMs with the terrestrial data is to bridge the spectral gap of the GOCE-based GGM functional as far as possible, so that the terrestrial observations are better approximated and the omission error does not obscure the comparisons [5]. In the SEM, the spectral gap between the GOCE-based GGMs and the terrestrial observations is bridged partially by a combination of: 1) the high-degree spectral bands of the EGM2008, and 2) omission error estimates sourced from RTM data. The latter completing signal, i.e. by adding the very short wavelength using the RTM (residual terrain modelling), can be computed from the Terrain Correction (TC) program developed by [23]. The GOCE-based models are expanded to a maximum d/o (e.g. Nmax) and then be compensated from the EGM2008 from $(\mathrm{Nmax}+1)$ up to 2190 . Beyond d/o 2190, the RTM omission error estimates are exploited to complete the spectral content of the model as best as possible. The following equation (9) describes the SEM strategy.

$$
\Delta g_{G G M}=\left.\Delta g_{G O C E(\text { point })}\right|_{2} ^{N_{\max }}+\left.\Delta g_{E G M 2008(\text { point })}\right|_{N_{\max +1}} ^{2190}+\Delta g_{T O P O}
$$

Subsequently, the residual gravity can be computed as

$$
\Delta g_{\text {res }}=\Delta g_{\text {terr }}-\Delta g_{G G M}
$$

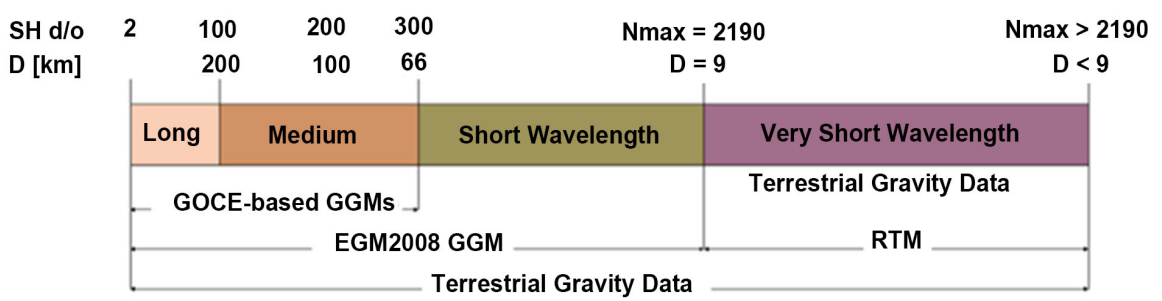

Figure 2. The Spectral consistency arrangement between GGMs and Terrestrial gravity data. 
Similarly, the geoid heights derived from the GOCE-based GGMs are evaluated with the corresponding ones obtained from the GPS/Levelling data at different spectral bands as

$$
\Delta N_{G G M}=\left.\Delta N\right|_{2} ^{N_{\max }}+\left.\Delta N\right|_{N_{\max +1}} ^{2190}+\Delta N_{\text {TOPO }}
$$

And the residual geoid heights between the GGMs and GPS/evelling read

$$
\Delta N_{\text {res }}=\Delta N_{G P S / \text { Levelling }}-\Delta N_{G G M}
$$

\section{Evaluation Results}

In the following sections we show the evaluation results based on using both GOCE-based models and ground-based data.

\subsection{Spectral Validation}

Figure 3 shows the differences of the degree variances in terms of geoid heights between the GOCE-based GGMs and the EGM2008 in a global scale and their corresponding cumulative geoid errors as given in Equation (3) and Equation (4), respectively. One can infer that there are obvious differences between the models from d/o 200 onward. The IGGT_R1 outperforms the other GOCEbased models particularly at the long wavelength of the gravity spectrum. The reason might bake to that the IGGT_R1 model uses a-priori gravity field information applied background model: EIGEN5C [29] up to d/o 240 which developed from high-resolution GRACE-based gravity field model. On the other hand, The SPW_R5 model shows a good behavior with respect to the other GGMs at the medium spectrum of the gravity due to that a-priori gravity field information have been used from the EIGEN-6C4 [30] and GOCO05C for signal covariance modelling. In addition, no corrections for SPW_R5 were computed to any gravity model (i.e. GOCE-only model). Moreover, cumulative geoid errors provided by the SPW_R5 model are less than those estimated from the IGGT_R1 and the other investigated models from d/o 200 onward.

Figure 4 depicts the differences of the degree variances in terms of gravity anomalies between the GOCE-based GGMs and the EGM2008 and their corresponding cumulative geoid errors as given in Equation (5) and Equation (6), respectively. Similarly, the IGGT_R1 and SPW_R5 models also provide an identical behaviour with respect to the other GGMs particularly at the long wavelength of the gravity spectrum as given by Figure 3 .

On a regional scale and for a reliability comparison between GOCE-based GGMs and the EGM2008 over the study area of Egypt, the investigated GOCE-based models are evaluated with the EGM2008 at the same maximum $\mathrm{d} / \mathrm{o}$ of their spherical harmonics at term of gravity anomalies and geoid heights. In this comparison, we have focused only on spherical harmonic from d/o 100 up to d/o 330 for two reasons: first, to test which of GOCE-based models approximates closely the gravity field over Egypt, particularly at the medium to short wavelength spectrum bands, where GOCE-based models contribution is 


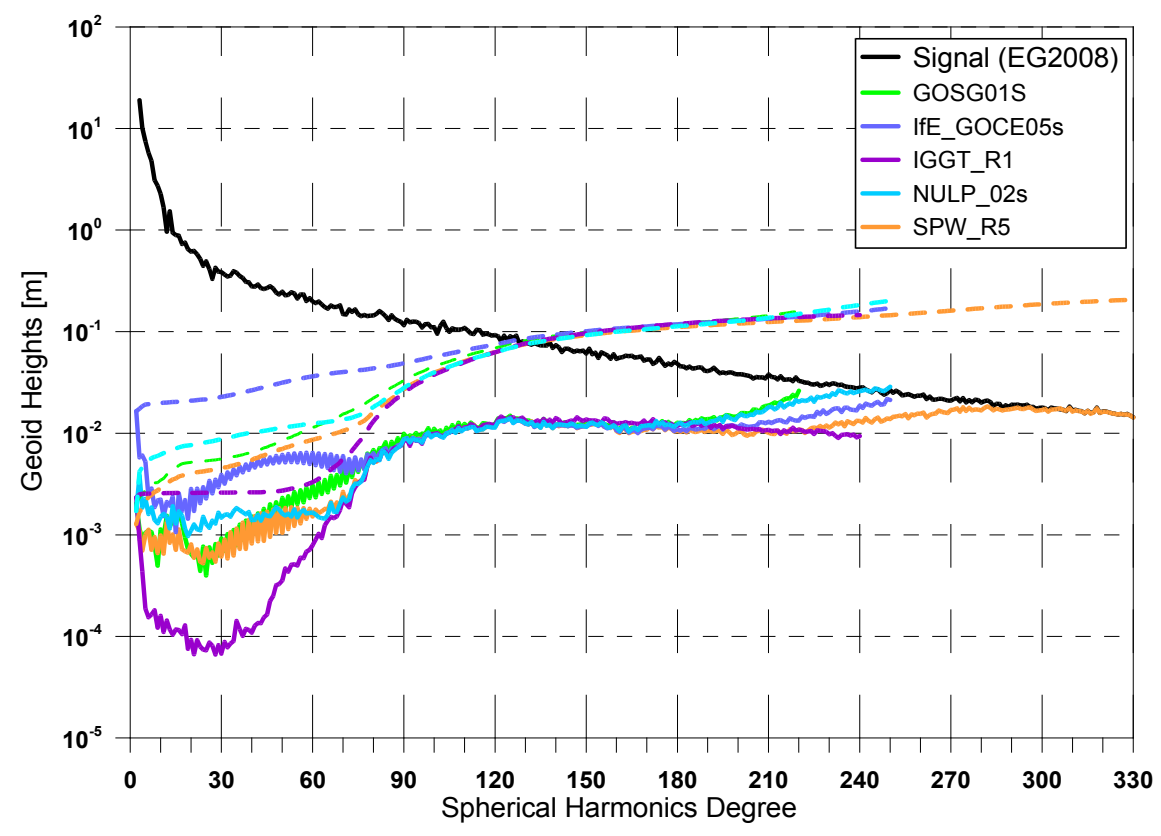

Figure 3. Difference degree variances in terms of geoid heights [m] between GOCE-based GGMs and the EGM2008 (solid-line) and their corresponding cumulative errors (dashed-line).

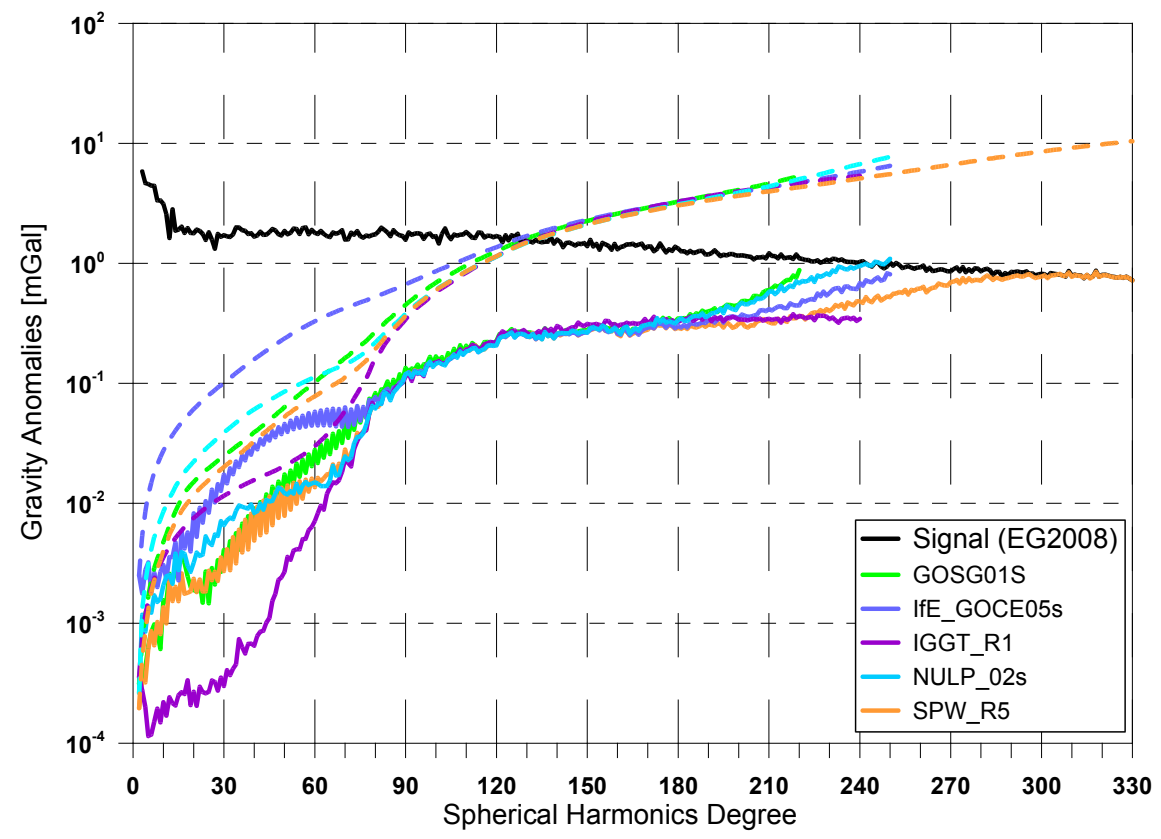

Figure 4. Difference Difference degree variances in terms of gravity anomalies [mgal] between GOCE-based GGMs and the EGM2008 (solid-line) and their corresponding cumulative errors (dashed-line).

significant. Second, to investigate at which spectral bands GOCE-based model deliver improved information. So, Figure 5 and Figure 6 show the results obtained from Equations (7) and (8) in term of Std of the differences between gravity anomalies and geoid heights, respectively. The IGGT_R1 model provides 


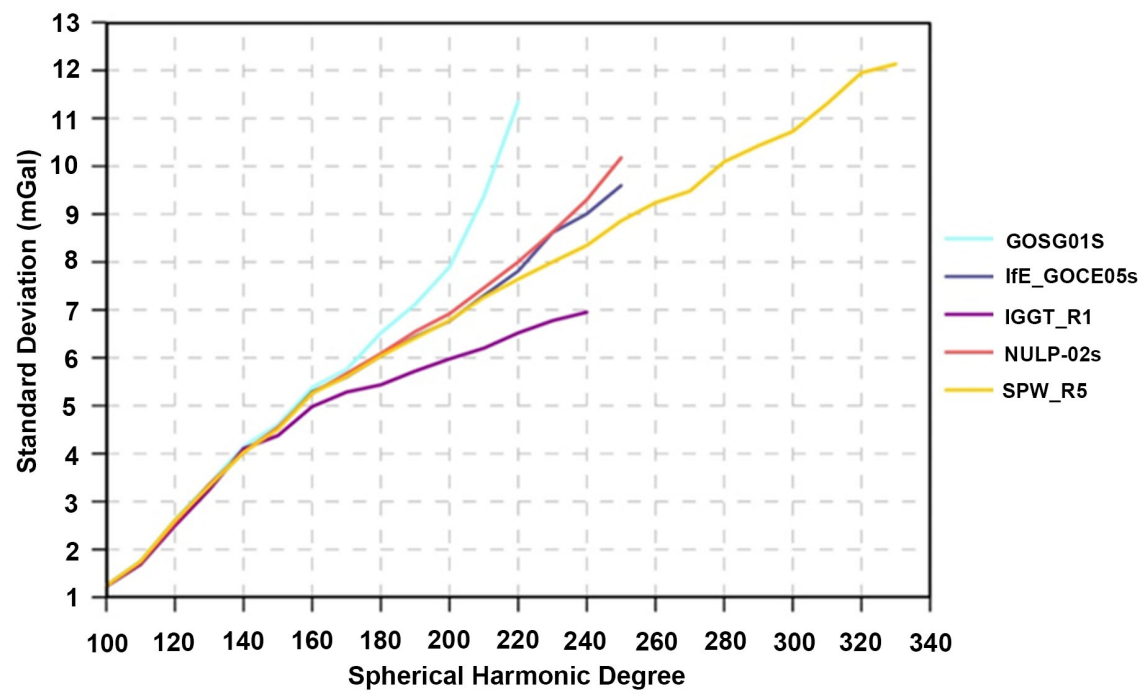

Figure 5. Std of the differences between free-air gravity anomalies in $\mathrm{mGal}$ obtained from GOCE-based GGMs and the corresponding ones calculated from the EGM2008 at (Nmax $=100,110,120, \cdots, 300)$.

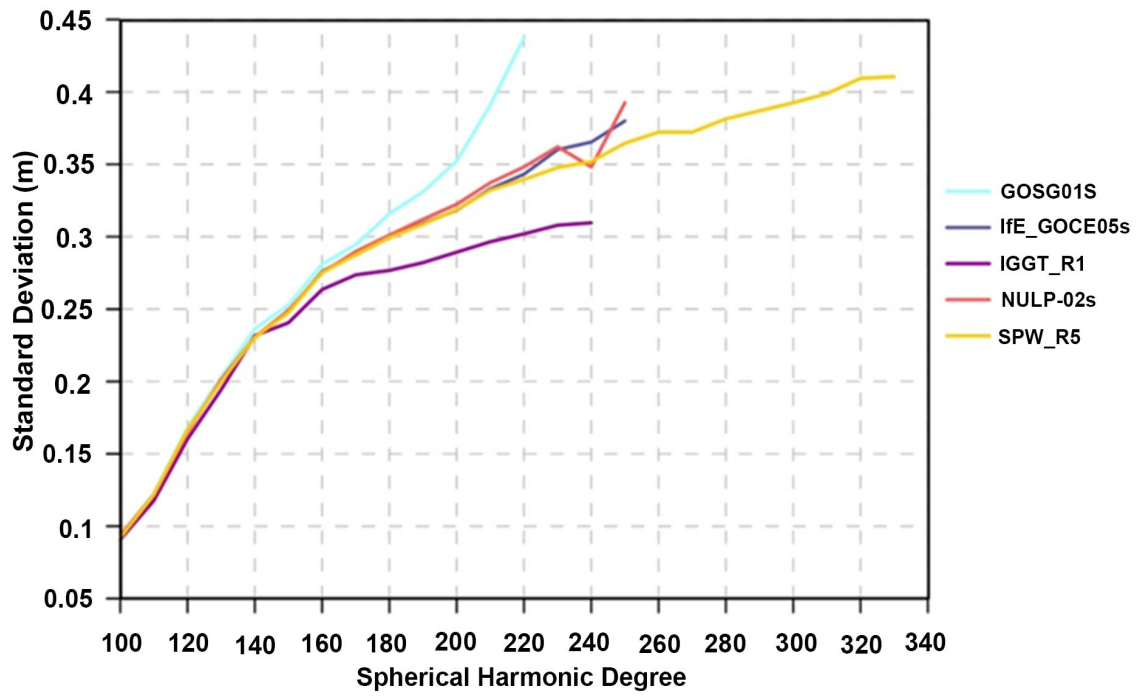

Figure 6. Std of the differences between geoid height in $\mathrm{m}$ obtained from GOCE-based GGMs and the corresponding ones calculated from the EGM2008 at (Nmax $=100,110$, $120, \cdots, 300)$.

the least differences from d/o 150 onward. This may due to the same reason mentioned before. Table 3 and Table 4 show the Std of the differences between free-air gravity anomalies in $\mathrm{mGal}$ and geoid heights in meters, respectively, as obtained from GOCE-based GGMs and the corresponding ones calculated from the EGM2008 at the same degree/order. The Std regarding GGMs based on GOCE-only satellite data for gravity anomalies and geoid heights are at the level of $5.43-6.52 \mathrm{mGal}$ and $0.276-0.315 \mathrm{~m}$ for d/o $180,5.977-7.89 \mathrm{mGal}$ and 0.289 - $0.352 \mathrm{~m}$ for d/o 200, $6.51-11.33 \mathrm{mGal}$ and $0.302-0.437 \mathrm{~m}$ for $\mathrm{d} / \mathrm{o} 220$, and $6.95-9.3 \mathrm{mGal}$ and $0.309-0.365 \mathrm{~m}$ for d/o 240 . The Std have increased rapidly 
Table 3. Statistic of the Std of the differences between free-air gravity anomalies in mGal obtained from GOCE-based GGMs and the corresponding ones calculated from the EGM2008 at $(\mathrm{Nmax}=100,110,120, \cdots, 300)$.

\begin{tabular}{|c|c|c|c|c|c|c|c|}
\hline Model name & $\begin{array}{c}\delta \Delta \mathrm{g} \\
(\mathrm{Nmax}=180) \\
\text { Std.[mGal] }\end{array}$ & $\begin{array}{c}\delta \Delta \mathrm{g} \\
(\mathrm{Nmax}=200) \\
\text { Std. }[\mathrm{mGal}]\end{array}$ & $\begin{array}{c}\delta \Delta \mathrm{g} \\
(\mathrm{Nmax}=220) \\
\text { Std. }[\mathrm{mGal}]\end{array}$ & $\begin{array}{c}\delta \Delta \mathrm{g} \\
(\mathrm{Nmax}=240) \\
\text { Std. }[\mathrm{mGal}]\end{array}$ & $\begin{array}{c}\delta \Delta \mathrm{g} \\
(\mathrm{Nmax}=260) \\
\text { Std. }[\mathrm{mGal}]\end{array}$ & $\begin{array}{c}\delta \Delta \mathrm{g} \\
(\mathrm{Nmax}=280) \\
\text { Std. }[\mathrm{mGal}]\end{array}$ & $\begin{array}{c}\delta \Delta \mathrm{g} \\
(\mathrm{Nmax}=300) \\
\text { Std. [mGall] }\end{array}$ \\
\hline NULP-02s & 6.08 & 6.92 & 8.00 & 9.30 & --------- & ------- & ------- \\
\hline SPW_R5 & 6.04 & 6.78 & 7.64 & 8.34 & 9.23 & 10.09 & 10.72 \\
\hline IfE_GOCE05s & 6.04 & 6.76 & 7.81 & 9.00 & --------- & --------- & --------- \\
\hline IGGT_R1 & 5.43 & 5.97 & 6.51 & 6.95 & -------- & -------- & -------- \\
\hline GOSG01S & 6.51 & 7.89 & 11.33 & --------- & --------- & --------- & -------- \\
\hline
\end{tabular}

Table 4. Statistic of the Std of the differences between geoid heights in m obtained from GOCE-based GGMs and the corresponding ones calculated from the EGM2008 at $(\mathrm{Nmax}=100,110,120, \ldots, 300)$.

\begin{tabular}{|c|c|c|c|c|c|c|c|}
\hline Model name & $\begin{array}{c}\delta \Delta \mathrm{N} \\
(\mathrm{Nmax}=180) \\
\text { Std. }[\mathrm{m}]\end{array}$ & $\begin{array}{c}\delta \Delta \mathrm{N} \\
(\mathrm{Nmax}=200) \\
\text { Std. }[\mathrm{m}]\end{array}$ & $\begin{array}{c}\delta \Delta \mathrm{N} \\
(\mathrm{Nmax}=220) \\
\text { Std. }[\mathrm{m}]\end{array}$ & $\begin{array}{c}\delta \Delta \mathrm{N} \\
(\mathrm{Nmax}=240) \\
\text { Std. }[\mathrm{m}]\end{array}$ & $\begin{array}{c}\delta \Delta \mathrm{N} \\
(\mathrm{Nmax}=260) \\
\text { Std. }[\mathrm{m}]\end{array}$ & $\begin{array}{c}\delta \Delta \mathrm{N} \\
(\mathrm{Nmax}=280) \\
\text { Std. }[\mathrm{m}]\end{array}$ & $\begin{array}{c}\delta \Delta \mathrm{N} \\
(\mathrm{Nmax}=300) \\
\text { Std. }[\mathrm{m}]]\end{array}$ \\
\hline NULP-02s & 0.301 & 0.322 & 0.348 & 0.348 & -------- & -------- & ------- \\
\hline SPW_R5 & 0.299 & 0.318 & 0.339 & 0.351 & 0.372 & 0.381 & 0.3922 \\
\hline IfE_GOCE05s & 0.299 & 0.318 & 0.343 & 0.365 & -------- & -------- & -------- \\
\hline IGGT_R1 & 0.276 & 0.289 & 0.302 & 0.309 & -------- & -------- & -------- \\
\hline GOSG01S & 0.315 & 0.352 & 0.437 & -.--.--.-. & --.---.--. & -.---.---. & ---------. \\
\hline
\end{tabular}

for all the GOCE-based GGMs solutions from d/o 200 onward because noise starts to dominate signals of GOCE [31].

\subsection{Results of Validation with Ground Gravity and GNSS/Levelling Data}

First, the residual gravity anomalies were computed from equation (10), which represents the difference between the gravity anomalies of GGMs after applying SEM and corresponding ones obtained from terrestrial gravity data. The results are shown in Figure 7 and their corresponding statistics are given in Table 5 in term of Std of the gravity anomalies differences. As seen in Figure 7, the least differences provided by the SPW_R5 are in rage of about $12.14 \mathrm{mGal}$ at SH d/o 230. Whereas, the Std of the gravity anomalies differences between the EGM2008 $(\mathrm{Nmax}=2190)$ and terrestrial gravity data are about $14.31 \mathrm{mGal}$. Second, the geoid heights obtained from ground GNSS/Levelling data have been compared with the corresponding ones derived from GOCE-based GGMs over the Egyptian region as given in Equation (12). The latter results are indicated in Figure 8 and their corresponding statistics are given in Table 6. As we can see in Figure 8, similarly the least differences are provided by the SPW_R5 of about 36.8 $\mathrm{cm}$ at SH d/o 280, while the Std of the geoid heights differences between the EGM2008 and GPS/Levelling $(\mathrm{Nmax}=2190)$ are about $54.3 \mathrm{~cm}$. Obviously, it can be concluded that the use of spectral enhancement method helped significantly 


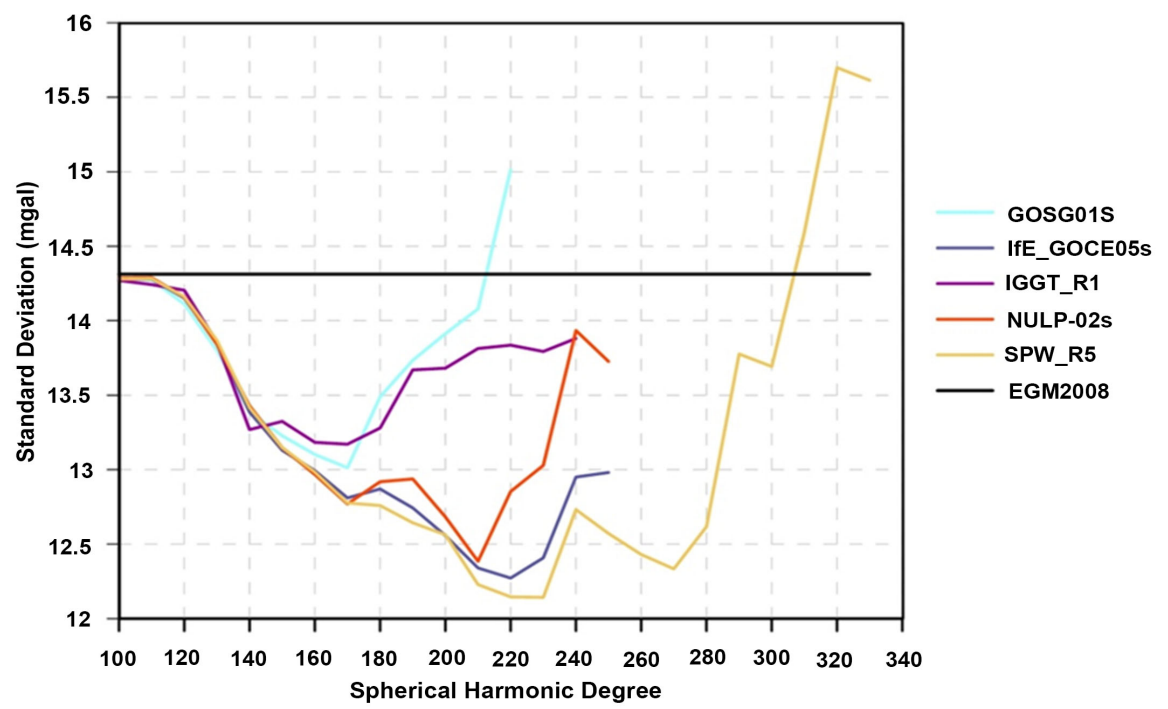

Figure 7. Std of the differences between the gravity anomaly obtained [mGal] from GOCE-based GGMs after applying the SEM and the corresponding ones from terrestrial gravity data.

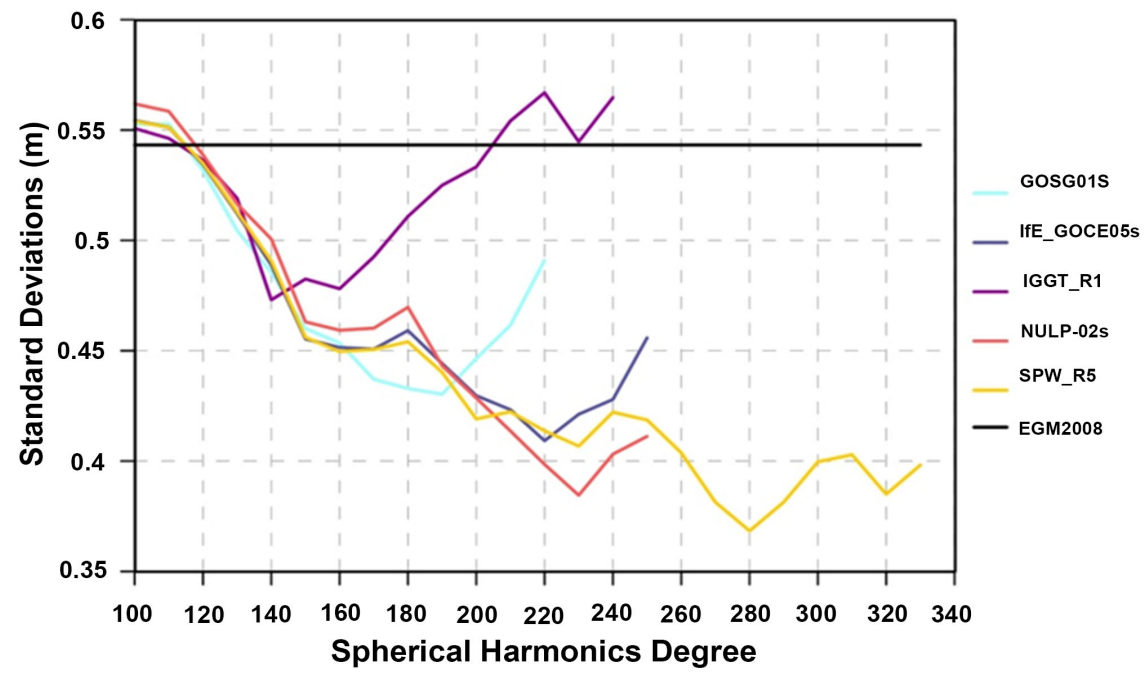

Figure 8. Std of the differences between the geoid heights obtained [m] from GOCE-based GGMs after applying the SEM and the corresponding ones from GPS/Levelling point.

Table 5. The standard deviation of the differences between GOCE-based GGMs and terrestrial gravity data in term of gravity anomaly ( $\mathrm{mGal}$ ) after applying the SEM.

\begin{tabular}{|c|c|c|c|c|c|c|c|}
\hline Model name & $\begin{array}{c}\delta \Delta \mathrm{g} \\
(\mathrm{Nmax}=180) \\
\text { Std.[mGal }]\end{array}$ & $\begin{array}{c}\delta \Delta \mathrm{g} \\
(\mathrm{Nmax}=200) \\
\text { Std. }[\mathrm{mGal}]\end{array}$ & $\begin{array}{c}\delta \Delta \mathrm{g} \\
(\mathrm{Nmax}=220) \\
\text { Std. [mGal] }\end{array}$ & $\begin{array}{c}\delta \Delta \mathrm{g} \\
(\mathrm{Nmax}=240) \\
\text { Std. }[\mathrm{mGal}]\end{array}$ & $\begin{array}{c}\delta \Delta \mathrm{g} \\
(\mathrm{Nmax}=260) \\
\text { Std. }[\mathrm{mGal}]\end{array}$ & $\begin{array}{c}\delta \Delta \mathrm{g} \\
(\mathrm{Nmax}=280) \\
\text { Std. }[\mathrm{mGal}]\end{array}$ & $\begin{array}{c}\delta \Delta \mathrm{g} \\
(\mathrm{Nmax}=300) \\
\text { Std. }[\mathrm{mGal}]]\end{array}$ \\
\hline NULP-02s & 12.91 & 12.68 & 12.85 & 13.93 & ------- & $-\cdots----$ & ------- \\
\hline SPW_R5 & 12.75 & 12.56 & 12.145 & 12.73 & 12.43 & 12.61 & 13.69 \\
\hline IfE_GOCE05s & 12.87 & 12.55 & 12.27 & 12.95 & -------- & -------- & -------- \\
\hline IGGT_R1 & 13.27 & 13.68 & 13.83 & 13.88 & --------- & -------- & -------- \\
\hline GOSG01S & 13.48 & 13.91 & 15.01 & -------- & -------- & -------- & -------- \\
\hline EGM2008 & 14.31 & 14.31 & 14.31 & 14.31 & 14.31 & 14.31 & 14.31 \\
\hline
\end{tabular}


Table 6. The standard deviation of the differences between GOCE-based GGMs and GPS/Levelling point in term of geoid height (m) after applying the SEM.

\begin{tabular}{|c|c|c|c|c|c|c|c|}
\hline Model name & $\begin{array}{c}\delta \Delta \mathrm{N} \\
(\mathrm{Nmax}=180) \\
\text { Std. }[\mathrm{m}]\end{array}$ & $\begin{array}{c}\delta \Delta \mathrm{N} \\
(\mathrm{Nmax}=200) \\
\text { Std. }[\mathrm{m}]\end{array}$ & $\begin{array}{c}\delta \Delta \mathrm{N} \\
(\mathrm{Nmax}=220) \\
\text { Std. }[\mathrm{m}]\end{array}$ & $\begin{array}{c}\delta \Delta \mathrm{N} \\
(\mathrm{Nmax}=240) \\
\text { Std. }[\mathrm{m}]\end{array}$ & $\begin{array}{c}\delta \Delta \mathrm{N} \\
(\mathrm{Nmax}=260) \\
\text { Std. }[\mathrm{m}]\end{array}$ & $\begin{array}{c}\delta \Delta \mathrm{N} \\
(\mathrm{Nmax}=280) \\
\text { Std. }[\mathrm{m}]\end{array}$ & $\begin{array}{c}\delta \Delta \mathrm{N} \\
(\mathrm{Nmax}=300) \\
\text { Std. }[\mathrm{m}]]\end{array}$ \\
\hline NULP-02s & 0.469 & 0.428 & 0.398 & 0.403 & --------- & --------- & --------- \\
\hline SPW_R5 & 0.454 & 0.419 & 0.413 & 0.422 & 0.403 & 0.368 & 0.399 \\
\hline IfE_GOCE05s & 0.459 & 0.429 & 0.409 & 0.427 & --------- & -------- & -------- \\
\hline IGGT_R1 & 0.510 & 0.533 & 0.566 & 0.564 & --------- & --------- & -------.- \\
\hline GOSG01S & 0.432 & 0.446 & 0.490 & -------- & -------- & -------- & -------- \\
\hline EGM2008 & 0.543 & 0.543 & 0.543 & 0.543 & 0.543 & 0.543 & 0.543 \\
\hline
\end{tabular}

in improving the quality of GOCE-based GGMs. A major reduction of about $15.16 \%$ in term of standard deviations of gravity anomaly differences and about $32.22 \%$ in terms of standard deviations of geoid heights differences are observed after applying the SEM by compensating the missing medium-to-short and very-short wavelength spectrum in GOCE-based GGMs by using the EGM2008 and the topographic information derived from the RTM method. So, the GOCE-based GGM of type SPW_R5 would be recommended to model geoid model for Egypt. This is a matter of our further investigation.

\section{Conclusions}

This paper investigated the evaluation of a number of latest releases of GOCEbased GGMs with the terrestrial gravity data and GPS/Levelling points over Egypt. In a first step, an evaluation on a global scale applying difference degree variances in terms of geoid heights and gravity anomalies and their cumulative errors between the studied GOCE-based GGMs and the EGM2008 was carried out. Subsequently, regional evaluations between GOCE-based GGMs and the EGM2008 on the one hand and between GOCE-based GGMs and the groundbased gravity and GNSS/Levelling data on the other hand have been performed. In the latter evaluation's type, the spectral consistency of both data frequency bands using the spectral enhancement method strategy has been accounted for.

Regarding to the evaluation process of GOCE-based GGMs with the EGM2008 over the Egyptian region, the findings show that the Std of the gravity anomaly anomalies and geoid heights differences are at the level of $5.43-6.52 \mathrm{mGal}$ and $0.276-0.315 \mathrm{~m}, 5.977-7.89 \mathrm{mGal}$ and $0.289-0.352 \mathrm{~m}, 6.51-11.33 \mathrm{mGal}$ and $0.302-0.437 \mathrm{~m}$, and $6.95-9.3 \mathrm{mGal}$ and $0.309-0.365 \mathrm{~m}$ for d/o 180, 200, 220 and 240, respectively. One can conclude that the IGGT_R1 model surpasses all investigated GGMs models at the previously mentioned degrees/orders, whereas from d/o 240 the SPW_R5 model outperforms the other GGMs.

Regarding to the evaluation process of GOCE-based GGMs with the gravity anomalies and geoid heights of the ground-based gravity and GNSS/Levelling data, one concludes that the use of the SEM helped much in improving the quality of the GOCE solutions. An obvious reduction of about $15.16 \%$ in term of 
standard deviations of gravity anomaly differences and about $32.22 \%$ in terms of Std of the geoid heights' differences could be achieved for the SPW_R5 model. This emphasizes that adding the significant part of the high-frequency gravity spectrum (i.e. the missing medium-to-short and very-short wavelength in GOCEbased GGMs) augmented by the extended EGM2008 and RTM provides valuable improvements for the validation purpose. Finally the GOCE-based GGM of type SPW_R5 is accordingly recommended to model geoid model for Egypt, which is a matter of our further investigation.

\section{Conflicts of Interest}

The authors declare no conflicts of interest regarding the publication of this paper.

\section{References}

[1] Pail, R., Goiginger, H., Mayrhofer, R., Schuh, W.-D., Brockmann, J.M., Krasbutter, I., Hoeck, E. and Fecher, T. (2010) GOCE Gravity Field Model Derived from Orbit and Gradiometry Data Applying the Time-Wise Method. The ESA Living Planet Symposium, Bergen, 28 June-2 July 2010.

[2] Bruinsma, S.L., Marty, J.C., Balmino, G., Biancale, R., Förste, C., Abrikosov, O. and Neumayer, H. (2010) GOCE Gravity Field Recovery by Means of the Direct Numerical Method. The ESA Living Planet Symposium, Bergen, 28 June-2 July 2010.

[3] Migliaccio, F., Reguzzoni, M., Sanso, F., Tscherning, C.C. and Veicherts, M. (2010) GOCE Data Analysis: The Space-Wise Approach and the First Space-Wise Gravity Field Model. ESA Publications Division, Norwijk, Bergen.

[4] Rummel, R., Gruber, T. and Koop, R. (2004) High Level Processing Facility for GOCE: Products and Processing Strategy. Proceedings of the 2nd International GOCE User Workshop "GOCE, the Geoid and Oceanography", Frascati, 8-10 March 2004, ESA SP-569.

[5] Hirt, C., Gruber, T. and Featherstone, W.E. (2011) Evaluation of the First GOCE Static Gravity Field Models Using Terrestrial Gravity, Vertical Deflections and EGM2008 Quasi Geoid Heights. Journal of Geodesy, 85, 723-740. https://doi.org/10.1007/s00190-011-0482-y

[6] Gruber, T., Visser, P.N.A.M., Ackermann, C. and Hosse, M. (2011) Validation of GOCE Gravity Field Models by Means of Orbit Residuals and Geoid Comparisons. Journal of Geodesy, 85, 845-860. https://doi.org/10.1007/s00190-011-0486-7

[7] Janák, J. and Pitonák, M. (2011) Comparison and Testing of GOCE Global Gravity Models in Central Europe. Journal of Geodetic Science, 1, 333-347. https://doi.org/10.2478/v10156-011-0010-2

[8] Guimarães, G. Matos, A. and Blitzkow, D. (2012) An Evaluation of Recent GOCE Geopotential Models in Brazil. Journal of Geodetic Science, 2, 144-155. https://doi.org/10.2478/v10156-011-0033-8

[9] Yi, W. and Rummel, R. (2014) A Comparison of GOCE Gravitational Models with EGM2008. Journal of Geodynamics, 73, 14-22. https://doi.org/10.1016/j.jog.2013.10.004

[10] Alothman, A., Bouman, J., Gruber, T., Lieb, V., Alsubaei, M., Alomar, A., Fuchs, M. and Schmidt, M. (2014) Validation of Regional Geoid Models for Saudi Arabia Using GPS/Levelling Data and GOCE Observations. IAG Symposia on Gravity, Geoid 
and Height Systems, Venice, 9-12 October 2014, Vol. 141, 193-200. https://doi.org/10.1007/978-3-319-10837-7_25

[11] Voigt, C. and Denker, H. (2014) Regional Validation and Combination of GOCE Gravity Field Models and Terrestrial Data. In: Flechtner, F., Sneeuw, N. and Schuh, W.-D., Eds., Observation of the System Earth from Space, Geotechnologien Science Report No. 20, Springer, Berlin, 139-145. https://doi.org/10.1007/978-3-642-32135-1_18

[12] Godah, W. and Krynski, J. (2015) Comparison of GGMs Based on One Year GOCE Observations with the EGM2008 and Terrestrial Data over the Area of Sudan. International Journal of Applied Earth Observation and Geoinformation, 35, 128-135. https://doi.org/10.1016/j.jag.2013.11.003

[13] Huang, J. and Veronneau, M. (2014) A Stokesian Approach for the Comparative Analysis of Satellite Gravity Models and Terrestrial Gravity Data. In: Marti, U., Ed., Gravity, Geoid and Height Systems, Springer, New York, Vol. 141, 101-107. https://doi.org/10.1007/978-3-319-10837-7_13

[14] Elsaka, B., Alothman, A. and Godah, W. (2015) On the Contribution of GOCE Satellite Based GGMs to Improve GNSS/Levelling Geoid Heights Determination in Saudi Arabia. IEEE Journal of Selected Topics in Applied Earth Observations and Remote Sensing, 9, 1-10. https://doi.org/10.1109/JSTARS.2015.2495193

[15] El-Ashquer, M., Elsaka, B. and El-Fiky, G. (2016) On the Accuracy Assessment of the Latest Releases of GOCE Satellite-Based Geopotential Models with EGM2008 and Terrestrial GPS/Levelling and Gravity Data over Egypt. International Journal of Geosciences, 7, 1323. https://doi.org/10.4236/ijg.2016.711097

[16] Pavlis, N.K., Holmes, S.A., Kenyon, S.C. and Factor, J.K. (2012) The Development and Evaluation of the Earth Gravitational Model 2008 (EGM2008). Journal of Geophysical Research, 117, B04406. https://doi.org/10.1029/2011JB008916

[17] Dawod, G. (1998) A National Gravity Standardization Network for Egypt. PhD Dissertation, Faculty of Engineering at Shoubra, Zagazig University, Zagazig.

[18] Marchenko, A., Marchenko, D. and Lopushansky, A. (2016) Gravity Field Models Derived from the Second Degree Radial Derivatives of the GOCE Mission: A Case Study. Annals of Geophysics, 59, s0649-s0659.

[19] Gatti, A., Reguzzoni, M., Migliaccio, F. and Sansò, F. (2016) Computation and Assessment of the Fifth Release of the GOCE-Only Space-Wise Solution. 1st Joint Commission 2 and IGFS Meeting, Thessaloníki, 19-23 September 2016.

[20] Wu, H., Müller, J. and Brieden, P. (2016) The IfE Global Gravity Field Model from GOCE-Only Observations. International Symposium on Gravity, Geoid and Height Systems, Thessaloníki, 19-23 September 2016.

[21] Lu, B., Luo, Z., Zhong, B., Zhou, H., Flechtner, F., Förste, C., Barthelmes, F. and Zhou, R. (2018) The Gravity Field Model IGGT_R1 Based on the Second Invariant of the GOCE Gravitational Gradient Tensor. Journal of Geodesy, 92, 561. https://doi.org/10.1007/s00190-017-1089-8

[22] Xu, X., Zhao, Y., Reubelt, T. and Robert, T. (2017) A GOCE Only Gravity Model GOSG01S and the Validation of GOCE Related Satellite Gravity Models. Geodesy and Geodynamics, 8, 260-272. https://doi.org/10.1016/j.geog.2017.03.013

[23] Forsberg, R. (1984) A Study of Terrain Reductions, Density Anomalies and Geophysical Inversion Methods in Gravity Field Modelling. Report 355, Department of Geodetic Science and Surveying, Ohio State University, Columbus. https://doi.org/10.21236/ADA150788

[24] Rapp, R.H. (1986) Global Geopotential Solutions. In: Sunkel, H., Ed., Mathematical 
and Numerical Techniques in Physical Geodesy, Springer-Verlag, Heidelberg.

[25] Rummel, R. and Van Gelderen, M. (1995) Meissl Scheme-Spectral Characteristics of Physical Geodesy. Manuscripta Geodaetica, 20, 379-385.

[26] Gruber, T. (2009) Evaluation of the EGM2008 Gravity Field by Means of GPS-Levelling and Sea Surface Topography Solutions.

[27] Voigt, C., Rülke, A., Denker, H., Ihde, J. and Liebsch, G. (2010) Validation of GOCE Products by Terrestrial Data Sets in Germany. Geotechnologien Science Report, 17, 106-111.

[28] Ihde, J., Wilmes, H., Müller, J., Denker, H., Voigt, C. and Hosse, M. (2010) Validation of Satellite Gravity Field Models by Regional Terrestrial Data Sets. In: Flechtner, F.M., et al., Eds., System Earth via Geodetic-Geophysical Space Techniques, Springer, Berlin, 277-296. https://doi.org/10.1007/978-3-642-10228-8_22

[29] Förste, C., Flechtner, F., Schmidt, R., Stubenvoll, R., Rothacher, M., Kusche, J., Neumayer, K.H., Biancale, R., Lemoine, J.M., Barthelmes, F., Bruinsma, S.L., König, R. and Meyer, U. (2008) EIGEN-GL05C: A New Global Combined High-Resolution GRACE-Based Gravity Field Model of the GFZ-GRGS Cooperation. Geophysical Research Abstracts, Vol. 10, Abstract No. EGU2008-A-06944, 2008, General Assembly European Geosciences Union, Vienna.

[30] Förste, C., Bruinsma, S., Abrykosov, O., Flechtner, F., Marty, J.-C., Lemoine, J.-M., Dahle, C., Neumayer, K.-H., Barthelmes, F., König, R. and Biancale, R. (2014) EIGEN-6C4: The Latest Combined Global Gravity Field Model Including GOCE Data up to Degree and Order 1949 of GFZ Potsdam and GRGS Toulouse. Geophysical Research Abstracts, Vol. 16, EGU2014-3707, General Assembly European Geosciences Union, Vienna.

[31] Rummel, R. (2010) GOCE: Gravitational Gradiometry in a Satellite. In: Freeden, W., Nashed, F.M.Z. and Sonar, T., Eds., Handbook of Geomathematics, Vol. 2, Springer, Berlin, 93-103. https://doi.org/10.1007/978-3-642-01546-5_4 